\title{
Walking activity during ambulant cardiac rehabilitation is related to maximum working capacity, age, and smoking behavior
}

This article was published in the following Dove Press journal: Vascular Health and Risk Management

\author{
Sinann AI Najem ${ }^{1,2}$ \\ Andreas Groll 3,4 \\ Axel Schmermund ${ }^{5,6}$ \\ Bernd Nowak ${ }^{5,6}$ \\ Thomas Voigtländer ${ }^{5,6}$ \\ Ulrike Kaltenbach ${ }^{5}$ \\ Peter Dohmann ${ }^{5}$ \\ Dietrich Andresen ${ }^{7}$ \\ Jürgen Scharhag ${ }^{2,8}$ \\ 'German Heart Foundation, \\ Frankfurt, Germany; ${ }^{2}$ Institute of \\ Sports and Preventive Medicine, \\ Saarbrücken, Germany; ${ }^{3}$ Chair of \\ Statistics, Georg August University, \\ Göttingen, Germany; ${ }^{4}$ Faculty of \\ Statistics, Technical University \\ Dortmund, Dortmund, Germany; \\ ${ }^{5}$ Cardioangiologisches Centrum \\ Bethanien Herzwerk, Frankfurt, \\ Germany; ${ }^{6}$ Bethanien Krankenhaus, \\ Cardioangiologisches Centrum \\ Bethanien, Frankfurt, Germany; \\ ${ }^{7}$ Lutheran Hospital Hubertus, Berlin, \\ Germany; ${ }^{8}$ Outpatients' Clinic for \\ Prevention, Rehabilitation and Sports \\ Medicine, Technical University of \\ Munich, Munich, Germany
}

Background: A total of 6,500 to 8,000 steps per day are recommended for cardiovascular secondary prevention. The aim of this research was to examine how many steps per day patients achieve during ambulant cardiac rehabilitation (CR), and if there is a correlation between the number of steps and physical and cardiological parameters.

Methods: In all, 192 stable CR patients were included and advised for sealed pedometry. The assessed parameters included maximum working capacity and heart rate, body mass index (BMI), New York Heart Association (NYHA) class, ejection fraction (EF), coronary artery disease status, beta-blocker medication, age, sex, smoking behavior, and laboratory parameters. A regularized regression approach called least absolute shrinkage and selection operator (LASSO) was used to detect a small set of explanatory variables associated with the response for steps per day. Based on these selected covariates, a sparse additive regression model was fitted.

Results: The model noted that steps per day had a strong positive correlation with maximum working capacity $(P=0.001)$, a significant negative correlation with higher age $(P=0.01)$ and smoking (smoker: $P<0.05$; ex-smoker: $P=0.01$ ), a positive correlation with high-density lipoprotein (HDL), and a negative correlation with beta-blockers. Correlation between BMI and walking activity was nonlinear (BMI 18.5-24: 7,427 $\pm 2,730$ steps per day; BMI 25-29:

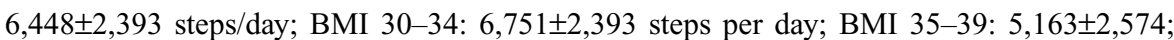
BMI >40: 6,077 $\pm 1,567)$.

Conclusion: Walking activity during CR is reduced in patients who are unfit, older, smoke, or used to smoke. In addition to training recommendations, estimated steps per day during CR could be seen as a baseline orientation that helps patients to stay generally active or even to increase activity after $\mathrm{CR}$.

Keywords: Step recommendation, steps/day in cardiac patients, pedometer

\section{Introduction}

The benefits of regular physical activity (PA) or fitness in healthy individuals or patients with cardiovascular disease are well established. ${ }^{1-5}$ Therefore, guidelines of medical associations suggest that healthy adults should have a minimum of 30 minutes of moderate activity five times a week, ${ }^{6,7}$ a minimum of 20 minutes of vigorous activity 3 days a week, or a combination of both ${ }^{6}$ with a minimal energy expenditure from the interval 500-1,000 metabolic equivalent of task (MET) - minutes per week. ${ }^{8}$ Activity should be performed continuously in minimal bouts of 10 minutes, and musculoskeletal activity is recommended twice a week. ${ }^{6}$ In secondary prevention, activity recommendations are similar, but individual risk determinants should be considered, especially in moderate- to high-risk patients, and training needs to be individually adjusted. ${ }^{9}$
Correspondence: Sinann AI Najem

German Heart Foundation,

Bockenheimer Landstraße 94-96, 60323

Frankfurt, Germany

Tel +4969955128144

Fax +4969955128345

Email alnajem@herzstiftung.de 
Cardiac rehabilitation (CR) promotes recovery and is an essential component of atherosclerotic cardiovascular disease management. ${ }^{10,11}$ Providing pedometers can help CR patients to increase their PA during and after rehabilitation. ${ }^{12,13}$ The recommendation of 10,000 steps per day for healthy people is commonly known. ${ }^{14,15}$ However, this recommendation could be too challenging for cardiac patients. ${ }^{16}$ Therefore, Ayabe et $\mathrm{al}^{17}$ compared the total amount of PA energy expenditure with the step activity in CR patients and suggested that a range of 6,500-8,500 steps per day would reach the generally recommended amount for secondary cardiovascular prevention. Houle et a $\mathrm{l}^{18}$ defined a target of $\geq 7,500$ steps per day to lower risk profile during 1 year after an acute coronary syndrome.

It is open to question whether a general step goal for cardiac patients is useful, since training needs to be individually adjusted. Pedometers are neither able to measure the frequency, intensity, or duration of person's PA bouts nor capable of capturing activities such as cycling, swimming, or resistance training. ${ }^{19}$ Furthermore, symptomatology in cardiac patients differs. Hence, the aims of this study were to analyze how many steps per day stable cardiac patients achieve during $\mathrm{CR}$ and to test if there is a correlation between the number of steps per day and physical and cardiological parameters.

\section{Methods}

\section{Subjects}

\section{Inclusion criteria}

All attendees were adult, cardiac-stable patients who had just started an ambulant CR (recruitment period: 1 year from July 2015 to July 2016) at Cardioangiologisches Centrum Bethanien (CCB), Herzwerk, Frankfurt. In general, CR lasts for 3 weeks ( 15 days, Monday to Friday). Compulsory attendance was 6 hours per day, including 2 hours of recovery time. CR consists of individualized and supervised PA training, psychosocial consultancy, lectures, and consultations to inform patients about their disease. Furthermore, classes to support healthy behaviors such as healthy eating, smoking cessation, and staying physically active were provided. Activity training focused on endurance (up to 30 minutes ergometry per day), strength (1 hour per day), gymnastic (three to four units of 1 hour per week), and coordination (1 hour, twice a week). In addition, little sport games and outdoor activities, such as hiking and Nordic walking, were offered. To avoid overwork, patients were told to rest over the weekend.
Patients with the following diseases were recruited:

- coronary heart disease \pm revascularization (recent percutaneous transluminal coronary angioplasty or coronary bypass);

- recent heart valve operation, reconstruction, or replacement;

- heart failure;

- severe hypertension; and

- post-pulmonary embolism.

After explaining the study design, a total of 280 patients were willing to participate and signed the consent form, which was approved by the institutional review board, the ethics committee of the Hessian Medical Association (Ärztekammer).

\section{Outcome measure}

\section{Medication}

Medication plans were used on admission day to categorize participants in terms of whether they were taking beta-blocker medication.

\section{Anthropometry and laboratory parameters}

Anthropometry (weight $[\mathrm{kg}]$, height $[\mathrm{cm}]$, and body mass index $[\mathrm{BMI}]$ ) and laboratory parameters (hemogram, cholesterol, high-density lipoprotein [HDL], low-density lipoprotein [LDL], creatinine, glomerular filtration rate, sodium, potassium, and blood glucose) were assessed in the morning on admission day. Fasting before blood collection was not necessary.

\section{Risk profile}

\section{Blood pressure and smoking behavior on admission day}

Systolic and diastolic blood pressure was assessed to be noninvasive with an upper arm blood pressure monitor (Boso Carat professional $^{\mathbb{}}$ ).

Smoking behavior was determined in order to categorize patients into "smoker" (0), "ex-smoker" (1), and "nonsmoker" (2). The pack-years of smokers and ex-smokers and the year that ex-smokers quit smoking were recorded.

\section{Exercise stress test}

Exercise testing via cycle ergometry is the most frequently used method in Europe. Therefore, every patient starting ambulant rehabilitation in the CCB Herzwerk underwent a symptom-limited exercise electrocardiogram on a bicycle ergometer (Watt $[\mathrm{W}]$ maximum, maximum heart rate, $\mathrm{W} / \mathrm{kg}$ 
bodyweight) to provide training control. Initial power output was $25 \mathrm{~W}$, followed by increases of $25 \mathrm{~W}$ every 2 minutes until patients could no longer maintain pedal cadence or indications for termination occurred. ${ }^{20}$

Ergometers from Ergoline Ergoselect $400^{\circledR}$ with monitors were used for testing and training (monitoring software: Ergoline ERS $2^{\circledR}$ ).

\section{New York Heart Association Class (NYHA)}

The admitting physician estimated NYHA based on patients' limitation during PA..$^{21}$

\section{Ejection fraction (EF)}

EF (generally calculated by Teichholz's formula) was assessed upon patients' admission by echocardiography using General Electric Vivid Pro $7^{\circledR}$; patients were categorized into four previously determined groups:

- $\mathrm{EF} 1:>55 \%$;

- $\mathrm{EF} 2:<55 \%-45 \%$;

- EF 3: $<45 \%-35 \%$; and

- $\mathrm{EF} 4:<35 \%$.

\section{Coronary artery disease status (concerned vessels)}

Patients were categorized into four previously determined groups. During coronary angiography, the interventional cardiologist assessed the level of stenosis by eyeballing:

- coronary artery disease 0 : no coronary artery stenosis $(>50 \%)$;

- coronary artery disease 1: stenosis in one main coronary artery $(>50 \%)$;

- coronary artery disease 2 : stenosis in two main coronary arteries (>50\%); and

- coronary artery disease 3: stenosis in three main coronary arteries $(>50 \%)$.

\section{PA assessment}

Patients received a sealed pedometer (Omron Walking Style Pro $2.0^{\circledR}$ ) to record habitual PA. It was sealed with black tape and programmed with the patient's height and weight. Patients were instructed to wear it the whole day, except when swimming, showering, and sleeping. For better compliance, patients were allowed to wear the pedometer in their pocket, attached with a clip on a belt, or like a necklace. Although pedometers are usually worn on the waist, chest wearing position provides accuracy as well. ${ }^{22}$ At the end of CR or at the latest after 21 days (memory time), step data were read out and patients were informed of their average steps per day during the entire period of rehabilitation.

Insignificant amounts of daily step data $(<100$ steps per day) were not counted in order to avoid a failure of misreading, such as when patients forgot their pedometer. The first day of step measurement and the day of discharge were also not counted, because patients did not wear the pedometer for a full day. Furthermore, patients were only included if they provided step data for $>50 \%$ of their CR stay (measured in days, excluding admission and discharging days).

\section{Statistical analyses}

To determine which of the present potential influential covariates had an effect on the step outcome, variable selection was performed using the least absolute shrinkage and selection operator (LASSO). ${ }^{23}$ The LASSO approach refers to an L1-penalization technique that applies a constraint on the sum of the regression coefficients' absolute values. Hence, coefficients are shrunk toward 0 , and less important effects can even be set to exactly 0 .

The strength of the penalization is controlled by the penalty parameter $\lambda$ : for large values of $\lambda$, only the coefficients of the most influential predictors are retained and all other effects are shrunk to 0 , whereas for lower values of $\lambda$, shrinkage is smaller and fewer coefficients are excluded from the model. Hence, the penalty parameter $\lambda$ plays the role of a tuning parameter and controls how many covariates are selected to be related to the response variable of the regression model. Usually, a rather high value for $\lambda$ is found to be optimal, and consequently, a sparse model with just a few relevant covariates is chosen. Thus, using the LASSO approach, implicit variable selection is normally achieved. In general, the major advantages of using the LASSO approach are that it is less affected by multicollinearity issues, reduces the prediction error, and leads to lower mean-squared errors. ${ }^{6}$

In order to account for categorical predictors such as NYHA or EF, an extended LASSO version was used that penalized the whole group of the dummy variables in correspondence with a single factor, namely, the group LASSO. ${ }^{24}$ Preliminary analyses revealed that the BMI nonlinearly affects the step outcome. Therefore, a potential polynomial effect of degree 4 was allowed for this variable. Altogether, we included the following 15 covariates into our group LASSO analysis: sex, age, smoking, BMI (polynomial with degree 4), coronary artery disease status, NYHA, EF, maximum 
working capacity ( $\mathrm{W} / \mathrm{kg}$ bodyweight), maximum heart rate, hemoglobin concentration, beta-blocker, hematocrit, cholesterol, HDL, and LDL.

The statistical analysis was conducted in $\mathrm{R}^{25}$ The group LASSO approach was implemented in the R-package grplasso, ${ }^{26}$ while the additive model was fitted using the mgcv package. ${ }^{27}$

\section{Results}

This section presents details on the exclusion of patients and detailed results of the final additive regression model (adjusted R-squared $=0.37$ ).

\section{Exclusion}

To ensure the capability of proper walking in our patients, we excluded 88 patients based on the following criteria after reviewing the health records:

- peripheral arterial disease $(\mathrm{n}=18)$;

- neurological or orthopedic relevant handicaps for walking: gonarthrosis, coxarthrosis $(n=19)$, and other relevant handicaps for walking $(n=4)$; and

- multiple exclusion criteria $(n=4)$ : combination of peripheral arterial obstructive diseases and neurological and/or orthopedic handicaps.

\section{Dropouts and missing data}

We had to dropout 21 patients because of CR or study abort and further 22 individuals because of missing or insufficient evaluable step data (technical problems or unwillingness to wear the pedometer during CR; one patient was too heavy for exercise electrocardiogram). As a result, the data of 192 patients were identified as eligibility for the study. Demographic data of these patients are presented in Table 1.

Out of the analyzed covariates, LASSO assigned nonzero effects to just a selection of six covariates: age, smoking, BMI (polynomial with degree 4), maximum working capacity (W/kg bodyweight), beta-blocker, and HDL. The coefficient estimates of the linear effects are presented in Table 2. All other variables were excluded from the model. The optimal penalty parameter $\lambda$ was determined via 10 -fold crossvalidation (Figure 1), and the corresponding coefficient paths are displayed in Figure 2.

Finally, an unregularized (post-LASSO) additive model was run on the selected set of six covariates to determine statistical significance and nonshrunk regression coefficient estimates. ${ }^{28}$ All covariates were included in the form of linear effects except for the BMI, which again was found to have a highly nonlinear effect (effective $d f: 4.983 ; P$-value: 0.191 ) on the step outcome (Figure 3). The detailed results for the remaining linear effects are summarized hereafter.

The major finding of this study is the strong, positive, and highly significant relationship between maximum working capacity (W/kg bodyweight), determined on admission (baseline), and steps per day in the whole CR period. Furthermore, for both age and smoking (reference level: never smoked),

Table I Patient group description (variable means together with their empirical SDs)

\begin{tabular}{|c|c|c|c|c|c|c|}
\hline & $\mathbf{n}$ & Steps/day & Working capacity & Age, years & BMI & Days in CRP \\
\hline Total & 192 & $6,530 \pm 2,504$ & $1.5 \pm 0.46$ & $59 \pm 11$ & $28.49 \pm 4.85$ & $24 \pm 5$ \\
\hline Male & 160 & $6,584 \pm 2,534$ & $1.56 \pm 0.45$ & $59 \pm I I$ & $28.49 \pm 4.54$ & $24 \pm 5$ \\
\hline Female & 32 & $6,257 \pm 2,365$ & $1.23 \pm 0.37$ & $58 \pm 10$ & $28.5 \pm 6.24$ & $26 \pm 4$ \\
\hline NYHAI & 139 & $6,817 \pm 2,598$ & $1.62 \pm 0.43$ & $58 \pm 10$ & $27.89 \pm 4.5 I$ & $24 \pm 5$ \\
\hline NYHA2 & 43 & $5,977 \pm 2,115$ & $1.23 \pm 0.35$ & $60 \pm 13$ & $29.09 \pm 5.01$ & $25 \pm 4$ \\
\hline NYHA3 & 10 & $4,9|7 \pm I, 74|$ & $0.97 \pm 0.3$ & $57 \pm 11$ & $34.3 \pm 4.86$ & $28 \pm 5$ \\
\hline EFI & 149 & $6,699 \pm 2,438$ & $1.52 \pm 0.43$ & $58 \pm 11$ & $28.62 \pm 4.92$ & $24 \pm 5$ \\
\hline EF2 & 28 & $6,374 \pm 2,994$ & $1.56 \pm 0.54$ & $62 \pm 10$ & $27.5 \pm 3.84$ & $24 \pm 4$ \\
\hline EF3 & 12 & $5,015 \pm 1,910$ & $1.17 \pm 0.47$ & $62 \pm 13$ & $29.42 \pm 6.2$ & $27 \pm 5$ \\
\hline EF4 & 3 & $5,638 \pm 667$ & $1.21 \pm 0.42$ & $62 \pm 12$ & $27.67 \pm 4.04$ & $23 \pm 4$ \\
\hline No coronary artery disease & 18 & $6,613 \pm 2,034$ & $1.48 \pm 0.4 \mathrm{I}$ & $5 I \pm 14$ & $28.94 \pm 5.12$ & $26 \pm 4$ \\
\hline Coronary artery disease I & 67 & $6,914 \pm 2,587$ & $1.58 \pm 0.46$ & $58 \pm I I$ & $28.12 \pm 5.62$ & $24 \pm 4$ \\
\hline Coronary artery disease 2 & 65 & $6,649 \pm 2,702$ & $1.54 \pm 0.42$ & $60 \pm 10$ & $28.7 I \pm 4.38$ & $24 \pm 5$ \\
\hline Coronary artery disease 3 & 42 & $5,697 \pm 2,085$ & $1.32 \pm 0.5$ & $62 \pm 10$ & $28.57 \pm 4.16$ & $25 \pm 5$ \\
\hline Normal weight: BMI I8.5-24 & 33 & $7,427 \pm 2,730$ & $1.74 \pm 0.45$ & $56 \pm 13$ & & $23 \pm 4$ \\
\hline Overweight: BMI 25-29 & 98 & $6,448 \pm 2,393$ & $1.59 \pm 0.43$ & $62 \pm 10$ & & $24 \pm 5$ \\
\hline Moderate obesity: BMI 30-34 & 36 & $6,75 I \pm 2,393$ & $1.34 \pm 0.36$ & $55 \pm 10$ & & $25 \pm 5$ \\
\hline Severe obesity: BMI 35-39 & 20 & $5,163 \pm 2,574$ & $1.05 \pm 0.31$ & $58 \pm 8$ & & $26 \pm 5$ \\
\hline Very severe obesity: $\mathrm{BMI}>40$ & 5 & $6,077 \pm 1,567$ & $1.05 \pm 0.14$ & $45 \pm 8$ & & $27 \pm 4$ \\
\hline
\end{tabular}

Abbreviations: BMI, body mass index; EF, ejection fraction; NYHA, New York Heart Association. 
Table 2 Estimated coefficients of the linear regression effects on steps/day obtained by an additive model with a nonlinear effect for BMI

\begin{tabular}{|l|l|l|l|l|}
\hline Parametric coefficients & Estimate & Standard error & $t$ value & $P(>[t])$ \\
\hline Intercept & $5,800.69$ & $1,512.5 I$ & 3.835 & $0.000173^{* * *}$ \\
\hline Age, years & -49.94 & $15.8 I$ & -3.185 & $0.001863^{* *}$ \\
\hline Smoker & -986.17 & 484.13 & -2.037 & $0.043113^{*}$ \\
\hline Ex-smoker & $-1,125.36$ & 344.97 & -3.262 & $0.001322^{* *}$ \\
\hline HDL & 15.47 & 12.09 & 1.280 & 0.202176 \\
\hline$W_{\max } /$ kg bodyweight & $2,623.7$ & 397.43 & 6.602 & $4.41 \mathrm{e}-10^{* * *}$ \\
\hline Beta-blocker medication & -277.7 & 316.82 & -0.877 & 0.381913 \\
\hline
\end{tabular}

Note: ${ }^{*}$-value $<0.05,{ }^{*} * P_{\text {-value }}<0.01$, and $* * * P$-value $<0.001$.

Abbreviations: BMI, body mass index; HDL, high-density lipoprotein; W, watt.

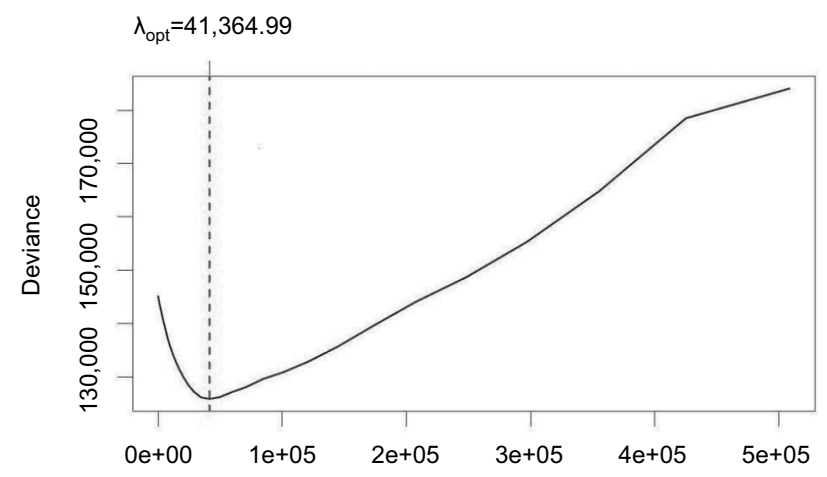

Figure I Optimal penalty parameter $\lambda$ has been determined via 10 -fold crossvalidation.

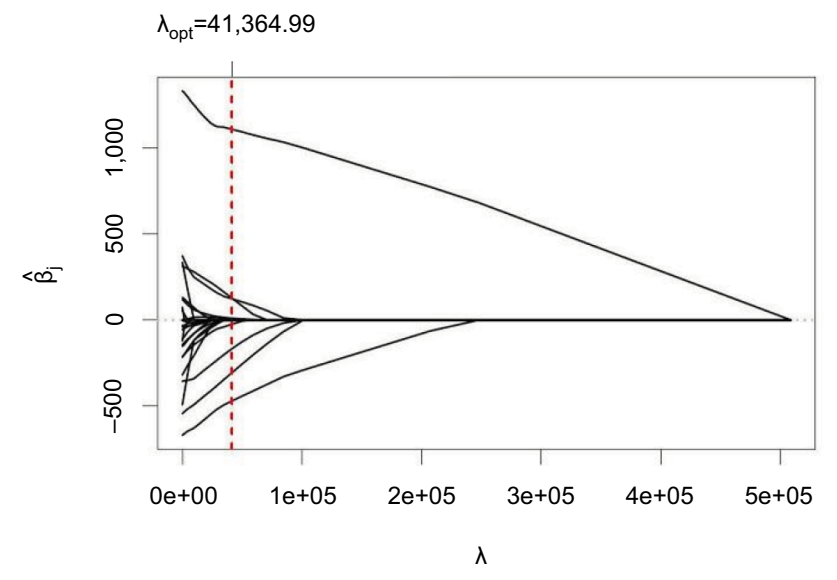

Figure 2 Coefficient paths vs the penalty parameter $\lambda$ (dashed vertical line: optimal penalty parameter $\lambda$ ).

significant negative relations were found. Finally, the model yielded a positive correlation for HDL and a negative correlation for beta-blocker on the step outcome, although both effects were only close to significance.

A nonlinear effect was found regarding BMI, which is displayed in Figure 1. Patients with a BMI between 30 and 35 exhibited increased step outcomes on average. The effect

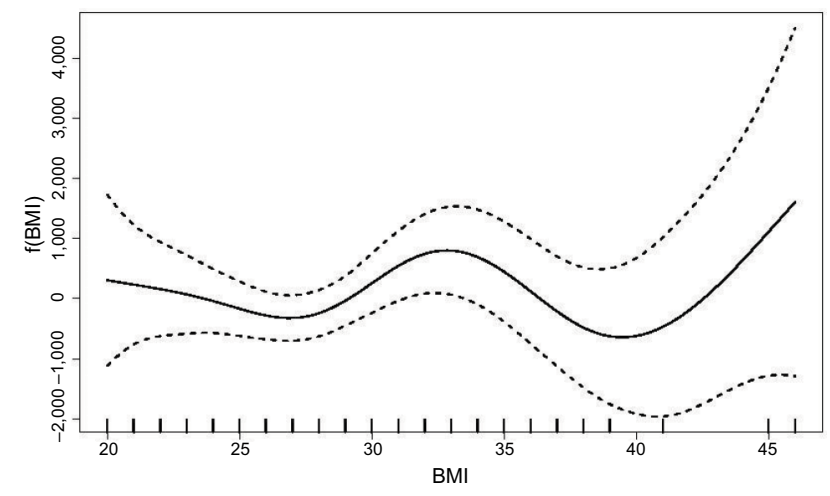

Figure 3 Nonlinear effect of BMI on step outcome. Abbreviation: BMI, body mass index.

decreased for higher BMI values and increased for very large BMI values. However, the wide point-wise significance bands indicate that this trend is uncertain due to very few observations with such high BMI values. Although the model's approximated $P$-value suggests nonsignificance, its overall fit in terms of adjusted R-squared strongly increases if the BMI is included with a nonlinear effect (for details on $P$-values for smooth effects, see $\mathrm{Wood}^{29}$ ).

The remaining set of variables, namely, NYHA, EF, coronary artery disease status, sex, maximum heart rate, hemoglobin, hematocrit, cholesterol, and LDL, were not selected by the variable selection approach and, therefore, were excluded from the final model. Consequently, these factors could not be directly related to the step outcome of patients during $\mathrm{CR}$.

Finally, although it was not a direct aim of the present study to analyze whether the general recommendation for CR patients to perform 10,000 steps per day is too challenging, we observed that most of the patients (89.6\%) were below this threshold. Only 20 patients, most of them part of the NYHA1 group ( $n=18$ ), reached a mean of $>10,000$ steps per day. 


\section{Discussion}

\section{Walking activity during CR}

The mean value of steps per day of the patients in our study was $6,530 \pm 2,504(n=192)$. This value is comparable to the outcome in the study of Ayabe et a $1^{17}$ (6,752 steps per day) and becomes closer if only NYHA I and NYHA II patients are studied (6,618 mean steps per day). Although exercise training in our CR did not only focus on walking activities mean steps per day of the patients in this study barely make it within the suggested range (6,500-8,000 steps per day) for cardiovascular secondary prevention. However, 99 (51.6\%) patients stayed below the threshold of 6,500 steps per day. Only 58 patients were above the threshold in the study of Houle et al, ${ }^{18}$ who advised for $>7,500$ steps per day to lower risk profile during 1 year after an acute coronary syndrome.

Although previous research tried to translate activity guidelines into step recommendations, pedometers could not determine intensity, duration, and type of activity. Therefore, it is difficult to set a general benchmark for steps per day for cardiac patients. ${ }^{19}$ Furthermore, it is obvious that fit patients will achieve more steps in 30 minutes of moderate jogging.

In our case, CR training was individualized (intensity, time), including cycling and resistance training. Walking activity in patients engaging in these activities will be reduced on training days. However, the number of steps per day provides an orientation of a person's general activity. ${ }^{19,30}$ In addition, step measurement offers an intuitive, readily understandable, accurate, and objective way to self-quantify PA. ${ }^{19}$ Therefore, it was assumed that the knowledge of the estimated steps per day during CR offers patients a useful orientation, especially after discharge. The findings reveal that the walking activity of patients during $\mathrm{CR}$ is essentially related to age, smoking behavior, BMI, HDL, maximum working capacity, and beta-blocker indication, with a nonlinear effect for BMI. However, only smoking behavior, age, and maximum working capacity were statistically significant. Although the average steps per day of male patients were higher than those of women, gender was not selected by the variable selection approach. All remaining factors were also not selected by the variable selection approach and, hence, could not be directly related to the step outcome of patients during CR.

\section{Working capacity}

Because of their strong relation, PA and fitness are often used to estimate prognosis in healthy individuals or patients with disease. ${ }^{1-5}$ As expected, the results of this study confirm the relationship of fitness, in this case measured in W/kg bodyweight (exercise electrocardiogram) and steps per day. The increase in 2,624 steps per day per W/kg bodyweight is high and attests that fitter patients walk more or that patients with a high walking activity are fitter.

\section{Age}

Aging is an unswayable risk factor, but cardiovascular risk age could differ. For example, a 40-year-old person with high levels of some risk factors may have the same risk as a 60-year-old person with ideal risk factor levels (nonsmoking, low level of cholesterol, ideal blood pressure) for an atherosclerotic event. ${ }^{31}$ Analogously, reduction in fitness or PA can be due to other factors than the aging process. It is possible to monitor a decrease of $\sim 500$ steps per day per decade. Hypothetically calculated and with all other variables kept constant, this decrease corresponds to a decrease in maximum working capacity of $0.19 \mathrm{~W} / \mathrm{kg}$ bodyweight, which seems to be easily stopped by a manageable training goal, especially in untrained patients.

\section{Smoking}

Smoking cessation is one of the most effective preventive measures in patients with coronary heart diseases and after myocardial infarction. ${ }^{32,33}$ Nonsmokers were more active than smokers ( -986 steps per day) or ex-smokers $(-1,125.36$ steps per day). There is some evidence that fitness $\left(\mathrm{VO}_{2} \max \right)$ is higher in nonsmokers than in persons with a smoking history. ${ }^{34}$ Furthermore, studies described an inverse association between smoking and health-related behaviors. ${ }^{35}$ As a result, it was assumed that impairment or unhealthy behaviors are responsible for the reduced walking activity.

However, it is more difficult to determine why former smokers walked less than patients who are still smoking. An explanation could be that on average, smokers in this study were younger than former smokers (smokers: 51 years, former smokers: 60 years, non-smokers: 61 years). Furthermore, it is important to note that 34 former smokers (total former smokers: 105 patients) stopped smoking during the year of $\mathrm{CR}$, potentially because of their cardiac issue. This factor should be considered to be a favorable behavior change, although it is not clear if those former smokers will stay permanent nonsmokers. It is possible that the adaption process concerning PA simply needs more time. The negative effect of smoking may decrease in the time spent not smoking.

\section{$\mathrm{BMI}$}

The smooth effect of BMI was not significant, but its approximated $P$-value was rather low, should have been noticed with respect to the adjusted R-squared. It was assumed that 
physically active patients have a high PA energy expenditure and therefore a low BMI. However, obese patients in our study walked more than patients with normal weight (compare Figure 1). This study does not have an explanation for this phenomenon, particularly because a higher fitness in the obese group could not be set (mean working capacity in patients with a BMI of 30-34.99: $1.34 \pm 0.36 \mathrm{~W} / \mathrm{kg}$ bodyweight, $\mathrm{n}=36$ vs. mean working capacity in patients with a BMI of 25-29.99: 1.59 $\pm 0.43 \mathrm{~W} / \mathrm{kg}$ bodyweight, $\mathrm{n}=98$ ).

\section{Beta-blocker}

It was observed that patients treated with beta-blockers walked less during CR. Although beta-blockers have been the standard care for patients with acute myocardial infarction, recently published studies question the utility of prolonged beta-blocker treatment in patients after acute myocardial infarction without heart failure or left ventricular dysfunction. ${ }^{36,37}$ In addition, the data suggest that beta-blockers may have a negative side effect on walking activity. However, statistical significance is missing. Hence, further discussion of whether every patient with myocardial infarction needs prolonged beta-blocker therapy or if therapy could have a negative effect on the exercise capacity would be without merit.

\section{HDL}

Low levels of HDL are a sign of increased cardiovascular risk. Even if medical therapy to increase HDL is out of the question, it is commonly known and accepted that lifestyleinduced HDL increase is strongly recommended.$^{38}$ There is some evidence that active acute coronary syndrome patients walking more than 7,500 steps per day for 12 months after hospitalization have higher HDL values. ${ }^{18}$ This study confirms this correlation in CR patients, admittedly with no statistical significance. In this case, an increase in 15 steps per day correlates with an increase of 1-unit HDL.

\section{Limitations}

These data should be used carefully. The outcome of the study is the results of participation in an ambulant CR in Frankfurt. It is unclear if step data during residential CR will differ or how step data will be shaped in cardiac patients without $\mathrm{CR}$ intervention. Of course, every CR will differ in terms of program. Some centers conduct more cycling activities; others may focus on walking or other activities. For better compliance, patients were advised to wear the pedometer all day long; therefore, the pedometer was worn during cycling activities as well. Furthermore, patients were allowed to wear the pedometer in different ways (pocket, attached on a belt, or like a necklace) that could have caused differences in step recording. In addition, it is important to notice that we examined the relationship between baseline measures, determined on admission day, and the average steps per day over the entire CR. Supposably, some of these measures may have changed over the period and the number of steps/day may have increased over time.

It should also be mentioned that the $P$-values and significance statements obtained from the final (unregularized) reestimation step succeeding the LASSO analysis should be regarded with caution. In general, these values and statements tend to underestimate the overall level of uncertainty in the estimates, as the uncertainty from the LASSO procedure is ignored.

\section{Conclusion}

This study examined the relationship between several cardiologically relevant parameters and walking activity (steps per day measured via pedometer) of patients ( $n=192)$ during CR. A specific characteristic of the present approach was the use of the modern variable selection technique LASSO. With this regularized regression approach, a small set of relevant explanatory variables for steps per day of $\mathrm{CR}$ patients could be detected. Based on these selected covariates, a sparse unregularized additive model was fitted to obtain unpenalized final regression coefficient estimates and to determine their statistical significance.

Our findings reveal that walking activity during ambulant CR was significantly reduced in patients who were unfit, older, smoked, or used to smoke (smokers and ex-smokers). Therefore, these parameters could be considered to estimate walking activity during CR. Furthermore, from the point of view that some activity is better than none and more is even better than some, ${ }^{39}$ the estimated steps per day during $\mathrm{CR}$ could be seen as a baseline orientation that helps patients to stay generally active or to even increase activity after CR. Follow-up research could clarify the development of steps per day after CR discharge in time, whether certain step counts have an impact on risk factors (blood pressure, weight), and how they accompany adverse events (re-hospitalization, cardiovascular events after CR, etc.).

\section{Acknowledgment}

This research has been financed by the German Heart Foundation. 


\section{Disclosure}

The authors report no conflicts of interest in this work.

\section{References}

1. Sattelmair J, Pertman J, Ding EL, Kohl HW 3rd, Haskell W, Lee IM. Dose response between physical activity and risk of coronary heart disease: a meta-analysis. Circulation. 2011;124(7):789-795.

2. Thompson PD, Buchner D, Pina IL, et al. Exercise and physical activity in the prevention and treatment of atherosclerotic cardiovascular disease: a statement from the Council on Clinical Cardiology (Subcommittee on Exercise, Rehabilitation, and Prevention) and the Council on Nutrition, Physical Activity, and Metabolism (Subcommittee on Physical Activity). Circulation. 2003;107(24):3109-3116.

3. Lee IM, Sesso HD, Oguma Y, Paffenbarger RS Jr. Relative intensity of physical activity and risk of coronary heart disease. Circulation. 2003;107(8):1110-1116.

4. Myers J, Prakash M, Froelicher V, Do D, Partington S, Atwood JE. Exercise capacity and mortality among men referred for exercise testing. N Engl J Med. 2002;346(11):793-801.

5. Blair SN, Kampert JB, Kohl HW 3rd, et al. Influences of cardiorespiratory fitness and other precursors on cardiovascular disease and all-cause mortality in men and women. JAMA. 1996;276(3):205-210.

6. Haskell WL, Lee IM, Pate RR, et al. Physical activity and public health: updated recommendation for adults from the American College of Sports Medicine and the American Heart Association. Circulation. 2007;116(9):1081-1093.

7. Pate RR, Pratt M, Blair SN, et al. Physical activity and public health. A recommendation from the Centers for Disease Control and Prevention and the American College of Sports Medicine. JAMA. 1995;273(5):402-407.

8. Garber CE, Blissmer B, Deschenes MR, et al; American College of Sports Medicine. American College of Sports Medicine position stand. Quantity and Quality of Exercise for Developing and Maintaining Cardiorespiratory, Musculoskeletal, and Neuromotor Fitness in Apparently Healthy Adults: guidance for prescribing exercise. Med Sci Sports Exerc. 2011;43(7):1334-1359.

9. Bjarnason-Wehrens B, Schulz O, Gielen S, et al. Leitlinie körperliche Aktivität zur Sekundärprävention und Therapie kardiovaskulärer Erkrankungen. Clin Res Cardiol Suppl. 2009;4(Supp13):1-44.

10. Kachur S, Chongthammakun V, Lavie CJ, et al. Impact of cardiac rehabilitation and exercise training programs in coronary heart disease. Prog Cardiovasc Dis. 2017;60(1):103-114.

11. Sandesara PB, Lambert CT, Gordon NF, et al. Cardiac Rehabilitation and Risk Reduction: time to "rebrand and reinvigorate". J Am Coll Cardiol. 2015;65(4):389-395.

12. Kaminsky LA, Jones J, Riggin K, Strath SJ. A pedometer-based physical activity intervention for patients entering a maintenance cardiac rehabilitation program: a pilot study. Cardiovasc Diagn Ther. 2013;3(2):73-79.

13. Butler L, Furber S, Phongsavan P, Mark A, Bauman A. Effects of a pedometer-based intervention on physical activity levels after cardiac rehabilitation: a randomized controlled trial. J Cardiopulm Rehabil Prev. 2009;29(2):105-114.

14. Bravata DM, Smith-Spangler C, Sundaram V, et al. Using pedometers to increase physical activity and improve health: a systematic review. JAMA. 2007;298(19):2296-2304.

15. Tudor-Locke C, Craig CL, Brown WJ, et al. How many steps/day are enough? For adults. Int J Behav Nutr Phys Act. 2011;8:79.

16. Tudor-Locke C, Craig CL, Aoyagi Y, et al. How many steps/day are enough? For older adults and special populations. Int J Behav Nutr Phys Act. 2011;8:80.

17. Ayabe M, Brubaker PH, Dobrosielski D, et al. Target step count for the secondary prevention of cardiovascular disease. Circ J. 2008;72(2):299-303.

18. Houle J, Valera B, Gaudet-Savard T, Auclair A, Poirier P. Daily steps threshold to improve cardiovascular disease risk factors during the year after an acute coronary syndrome. J Cardiopulm Rehabil Prev. 2013;33(6):406-410.
19. Bassett DR Jr, Toth LP, LaMunion SR, Crouter SE. Step Counting: A Review of Measurement Considerations and Health-Related Applications. Sports Med. 2017;47(7):1303-1315.

20. Fletcher GF, Ades PA, Kligfield P, et al; American Heart Association Exercise, Cardiac Rehabilitation, and Prevention Committee of the Council on Clinical Cardiology, Council on Nutrition, Physical Activity and Metabolism, Council on Cardiovascular and Stroke Nursing, and Council on Epidemiology and Prevention. Exercise standards for testing and training: a scientific statement from the American Heart Association. Circulation. 2013;128(8):873-934.

21. Dolgin M. Nomenclature and Criteria for Diagnosis of Diseases of the Heart and Great Vessels: The Criteria Committee of the New York Heart Association. 9th ed. Boston, MA: Little Brown Handbook; 1994.

22. Park W, Lee VJ, Ku B, Tanaka H. Effect of walking speed and placement position interactions in determining the accuracy of various newer pedometers. J Exerc Sci Fit. 2014;12(1)31:37.

23. Tibshirani R. Regression Shrinkage and selection via the Lasso. $J R$ Statist Soc B. 1996;58(1):267-288.

24. Meier L, van de Geer S, Bühlmann P. The group lasso for logistic regression. J R Statist Soc B. 2008;70(1):53-71.

25. $\mathrm{R}$ Core Team [homepage on the Internet]. R: A Language and Environment for Statistical Computing. Vienna, Austria; 2016. Available from: https://www.R-project.org/. Accessed March 21, 2018.

26. Meier L. grplasso: Fitting user specified models with Group Lasso penalty. R package (version 0.4-5); 2015.

27. Wood SN. Generalized Additive Models: An Introduction With R. Boca Raton: Chapman \& Hall/CRC; 2006.

28. Wood SN. Fast stable restricted maximum likelihood and marginal likelihood estimation of semiparametric generalized linear models. $J$ R Statist Soc Series B. 2011;73(1):3-36.

29. Wood SN. On p-values for smooth components of an extended generalized additive model. Biometrika. 2013;100(1):221-228.

30. Tudor-Locke C, Bassett DR Jr. How many steps/day are enough? Preliminary pedometer indices for public health. Sports Med. 2004;34(1):1-8.

31. Piepoli MF, Hoes AW, Agewall S, et al; ESC Scientific Document Group. 2016 European Guidelines on cardiovascular disease prevention in clinical practice: The Sixth Joint Task Force of the European Society of Cardiology and Other Societies on Cardiovascular Disease Prevention in Clinical Practice (constituted by representatives of 10 societies and by invited experts)Developed with the special contribution of the European Association for Cardiovascular Prevention \& Rehabilitation (EACPR). Eur Heart J. 2016;37(29):2315-2381.

32. Critchley J, Capewell S. Smoking cessation for the secondary prevention of coronary heart disease. Cochrane Database Syst Rev. 2004;(1):CD003041.

33. Chow CK, Jolly S, Rao-Melacini P, Fox KA, Anand SS, Yusuf S. Association of diet, exercise, and smoking modification with risk of early cardiovascular events after acute coronary syndromes. Circulation. 2010;121(6):750-758.

34. de Borba AT, Jost RT, Gass R, et al. The influence of active and passive smoking on the cardiorespiratory fitness of adults. Multidiscip Respir Med. 2014;9(1):34.

35. Wang M, Wang H, Fei FR, Xu CX, Du XF, Zhong JM. The associations between cigarette smoking and health-related behaviors among Chinese school-aged adolescents. Tob Induc Dis. 2017;15:27.

36. Dondo TB, Hall M, West RM, et al. $\beta$-Blockers and Mortality After Acute Myocardial Infarction in Patients Without Heart Failure or Ventricular Dysfunction. J Am Coll Cardiol. 2017;69(22):2710-2720.

37. Puymirat E, Riant E, Aissaoui N, et al. $\beta$ blockers and mortality after myocardial infarction in patients without heart failure: multicentre prospective cohort study. BMJ. 2016;354:i4801.

38. März W, Kleber ME, Scharnagl H, et al. Klinische Bedeutung des HDL-Cholesterins [Clinical importance of HDL cholesterol]. Herz. 2017;42(1):58-66. German.

39. Powell KE, Paluch AE, Blair SN. Physical activity for health: What kind? How much? How intense? On top of what? Annu Rev Public Health. 2011;32:349-365. 


\section{Publish your work in this journal}

Vascular Health and Risk Management is an international, peerreviewed journal of therapeutics and risk management, focusing on concise rapid reporting of clinical studies on the processes involved in the maintenance of vascular health; the monitoring, prevention and treatment of vascular disease and its sequelae; and the involvement of metabolic disorders, particularly diabetes. This journal is indexed on PubMed Central and MedLine. The manuscript management system is completely online and includes a very quick and fair peer-review system, which is all easy to use. Visit http://www.dovepress.com/ testimonials.php to read real quotes from published authors.

Submit your manuscript here: https://www.dovepress.com/vascular-health-and-risk-management-journal 